\title{
Patients with IDH1 wild type anaplastic astrocytomas exhibit worse prognosis than IDH1-mutated glioblastomas, and IDH1 mutation status accounts for the unfavorable prognostic effect of higher age: implications for classification of gliomas
}

\author{
Hartmann, C ; Hentschel, B ; Wick, W ; Capper, D ; Felsberg, J ; Simon, M ; Westphal, M ; Schackert, \\ G ; Meyermann, R ; Pietsch, T ; Reifenberger, G ; Weller, M ; Loeffler, M ; von Deimling, A
}

\begin{abstract}
WHO grading of human brain tumors extends beyond a strictly histological grading system by providing a basis predictive for the clinical behavior of the respective neoplasm. For example, patients with glioblastoma WHO grade IV usually show a less favorable clinical course and receive more aggressive first-line treatment than patients with anaplastic astrocytoma WHO grade III. Here we provide evidence that the IDH1 status is more prognostic for overall survival than standard histological criteria that differentiate high-grade astrocytomas. We sequenced the isocitrate dehydrogenase 1 gene (IDH1) at codon 132 in 382 patients with anaplastic astrocytoma and glioblastoma from the NOA-04 trial and from a prospective translational cohort study of the German Glioma Network. Patients with anaplastic astrocytomas carried IDH1 mutations in $60 \%$, and patients with glioblastomas in $7.2 \%$. IDH1 was the most prominent single prognostic factor (RR 2.7; 95\% CI 1.6-4.5) followed by age, diagnosis and MGMT. The sequence from more favorable to poorer outcome was (1) anaplastic astrocytoma with IDH1 mutation, (2) glioblastoma with IDH1 mutation, (3) anaplastic astrocytoma without IDH1 mutation and (4) glioblastoma without IDH1 mutation $(\mathrm{p}<0.0001)$. In this combined set of anaplastic astrocytomas and glioblastomas both, IDH1 mutation and IDH1 expression status were of greater prognostic relevance than histological diagnosis according to the current WHO classification system. Our data indicate that much of the prognostic significance of patient age is due to the predominant occurrence of IDH1 mutations in younger patients. Immunohistochemistry using a mutation-specific antibody recognizing the $\mathrm{R} 132 \mathrm{H}$ mutation yielded similar results. We propose to complement the current WHO classification and grading of high-grade astrocytic gliomas by the IDH1 mutation status and to use this combined histological and molecular classification in future clinical trials.
\end{abstract}

DOI: https://doi.org/10.1007/s00401-010-0781-z

Posted at the Zurich Open Repository and Archive, University of Zurich

ZORA URL: https://doi.org/10.5167/uzh-43783

Journal Article

Accepted Version

Originally published at:

Hartmann, C; Hentschel, B; Wick, W; Capper, D; Felsberg, J; Simon, M; Westphal, M; Schackert, G; Meyermann, R; Pietsch, T; Reifenberger, G; Weller, M; Loeffler, M; von Deimling, A (2010). Patients with IDH1 wild type anaplastic astrocytomas exhibit worse prognosis than IDH1-mutated glioblastomas, 
and IDH1 mutation status accounts for the unfavorable prognostic effect of higher age: implications for classification of gliomas. Acta Neuropathologica, 120(6):707-718.

DOI: https://doi.org/10.1007/s00401-010-0781-z 


\section{Patients with IDH1 wild type anaplastic astrocytomas exhibit}

worse prognosis than IDH1 mutated glioblastomas and IDH1

mutation status accounts for the unfavorable prognostic effect

of higher age: implications for classification of gliomas

Christian Hartmann ${ }^{1,2}$, Bettina Hentschel ${ }^{3}$, Wolfgang Wick ${ }^{4}$, David Capper ${ }^{1}$, Jörg Felsberg ${ }^{5}$, Matthias Simon $^{6}$, Manfred Westphal ${ }^{7}$, Gabriele Schackert $^{8}$, Richard Meyermann ${ }^{9}$, Torsten Pietsch ${ }^{10}$, Guido Reifenberger ${ }^{5}$, Michael Weller ${ }^{11}$, Markus Loeffler ${ }^{3}$, Andreas von Deimling $^{1,2}$

${ }^{1}$ Department of Neuropathology, Institute of Pathology, Ruprecht-Karls-University Heidelberg, Im Neuenheimer Feld 220/221, D-69120 Heidelberg, Germany

${ }^{2}$ Clinical Cooperation Unit Neuropathology, German Cancer Research Center, D-69120 Heidelberg,

Germany

${ }^{3}$ Institute for Medical Informatics, Statistics and Epidemiology, Universität Leipzig, Härtelstr. 16-18, D04107 Leipzig, Germany

${ }^{4}$ Department of Neurooncology, Neurology, Ruprecht-Karls-University Heidelberg, Im Neuenheimer Feld 400, D-69120 Heidelberg, Germany, and, Clinical Cooperation Unit Neurooncology, German Cancer Research Center, 69120 Heidelberg, Germany

${ }^{5}$ Department of Neuropathology, Heinrich Heine University, Moorenstr. 5, D-40225 Düsseldorf, Germany

${ }^{6}$ Department of Neurosurgery, University of Bonn, Sigmund Freud Str. 25, D-53105 Bonn, Germany

${ }^{7}$ Department of Neurosurgery, University Hamburg-Eppendorf, Martinistr. 52, D-20251 Hamburg, Germany

${ }^{8}$ Department of Neurosurgery, University Dresden, Fetscherstr. 74, D-01307 Dresden, Germany 
${ }^{9}$ Brain Research Institute, Eberhard Karls-University Tübingen, Calwerstr. 3, D-72076 Tübingen, Germany

${ }^{10}$ Department of Neuropathology, University of Bonn, Siegmund-Freud-Str. 25, D-53105 Bonn, Germany

${ }^{11}$ Department of Neurology, University Hospital Zurich, Frauenklinikstr. 26, CH-8091 Zurich, Switzerland

\section{Corresponding Author}

Prof. Dr. med. Andreas von Deimling

Ruprecht-Karls-Universität Heidelberg

Department of Neuropathology

Institute of Pathology

Clinical Cooperation Unit Neuropathology

German Cancer Research Center

Im Neuenheimer Feld 220/221

D-69120 Heidelberg

Fon: $\quad+49(0) 6221562603 / 2604$

Fax: $\quad+49(0) 6221564566$

Email: andreas.vondeimling@med.uni-heidelberg.de

Running title

IDH1 mutation and classification of high grade gliomas 


\section{Abstract}

WHO grading of human brain tumors extends beyond a strictly histological grading system by providing a basis predictive for the clinical behavior of the respective neoplasm. For example, patients with glioblastoma WHO grade IV usually show a less favorable clinical course and receive more aggressive first-line treatment than patients with anaplastic astrocytoma WHO grade III. Here we provide evidence that the IDH1 status is more prognostic for overall survival than standard histological criteria that differentiate high-grade astrocytomas. We sequenced the isocitrate dehydrogenase 1 gene (IDH1) at codon 132 in 382 patients with anaplastic astrocytoma and glioblastoma from the NOA-04 trial and from a prospective translational cohort study of the German Glioma Network. Patients with anaplastic astrocytomas carried IDH1 mutations in $60 \%$, and patients with glioblastomas in $7.2 \%$. IDH1 was the most prominent single prognostic factor $(\mathrm{RR}=2.7 ; 95 \%-\mathrm{Cl} 1.6$ to 4.5$)$ followed by age, diagnosis and MGMT. The sequence from more favorable to poorer outcome was 1) anaplastic astrocytoma with IDH1 mutation, 2) glioblastoma with IDH1 mutation, 3) anaplastic astrocytoma without IDH1 mutation and 4) glioblastoma without IDH1 mutation $(\mathrm{p}<0.0001)$. In this combined set of anaplastic astrocytomas and glioblastomas both, IDH1 mutation and IDH1 expression status were of greater prognostic relevance than histological diagnosis according to the current WHO classification system. Our data indicate that much of the prognostic significance of patient age is due to the predominant occurrence of $I D H 1$ mutations in younger patients. Immunohistochemistry using a mutation-specific antibody recognizing the $\mathrm{R} 132 \mathrm{H}$ mutation yielded similar results. We propose to complement the current WHO classification and grading of high-grade astrocytic gliomas by the IDH1 mutation status and to use this combined histological and molecular classification in future clinical trials.

Keywords: grading; classification; anaplastic astrocytoma; glioblastoma; IDH1 mutation; MGMT; age; immunohistochemistry; prognosis 


\section{Introduction}

WHO classification and grading of human brain tumors is a dynamic classification system undergoing regular updating. Classification of brain tumors relies on the presumed recognition of cell lineages giving rise to specific tumor entities. WHO classification of brain tumors distinguishes diffuse astrocytomas and glioblastomas among astrocytic tumors on grounds of their predominant astrocytic differentiation. Grading of astrocytomas relies on an evaluation of anaplasia, dedifferentiation and malignancy. The WHO criteria for anaplasia in diffuse astrocytomas include increased cellularity, distinct nuclear atypia and mitotic activity. Glioblastoma are defined as anaplastic, cellular gliomas composed of poorly differentiated, often pleomorphic astrocytic tumor cells with marked nuclear atypia and brisk mitotic activity. Prominent microvascular proliferation and/or necrosis are essential diagnostic features. Malignancy, however, extends beyond the term of anaplasia because it evokes clinical parameters such as tumor recurrence and clinical outcome. The WHO classification of brain tumors has emphasized a malignancy scale underlying its brain tumor grading rather than a purely histology based algorithm [11]. Thus, the identification of glioma subgroups exhibiting significant differences in clinical outcome within an established WHO tumor entity would necessitate an adjustment of the WHO grading system. At present the distinction between anaplastic astrocytoma and glioblastoma has clinical implications regarding the choice of treatments with more aggressive treatments for glioblastoma. Hence, novel prognostic factors may influence clinical decision making regarding treatment.

Cytosolic isocitrate dehydrogenase 1 (IDH1) mutations were initially detected in a fraction of glioblastomas [14] followed by the observation that they are present in the majority of diffuse astrocytomas, oligodendrogliomas and mixed oligoastrocytomas of WHO grades II and III [1, 7, 9, 22, 26]. Consistently, the IDH1 mutation rate is also high in secondary glioblastoma developing from previously diagnosed astrocytoma. While approximately $70 \%$ of the diffuse astrocytomas and secondary glioblastomas carry IDH1 mutations, this alteration is observed in less than $10 \%$ of primary glioblastoma. These observations so far provide the strongest molecular evidence for different origins of primary glioblastoma and secondary glioblastoma [13]. On the other hand, the occurrence of IDH1 mutations in the majority of both diffuse astrocytomas and oligodendroglial tumors requires reevaluation of the relation between these tumor entities. This contrasts findings suggesting a separation of astrocytic and oligodendroglial tumors on grounds of characteristic genomic alterations, 
mainly TP53 mutations in diffuse astrocytoma and combined 1p/19q losses in oligodendroglial tumors [10]. These observations indicate that $I D H 1$ mutational status may be a marker distinguishing tumor lineages and point to a common origin of diffuse astrocytoma including secondary glioblastomas and oligodendroglial tumors. Determination of $I D H 1$ mutation frequency in these tumors was followed by clinical analyses revealing that this mutation constitutes a favorable prognostic factor for both patients with anaplastic astrocytoma [25] and glioblastoma [23]. In fact, the prognostic power of presence or absence of this mutation exceeded that of other markers such as MGMT promoter methylation status.

The role of $I D H 1$ mutations in tumorigenesis is under intense investigation. These mutations, which nearly always affect codon 132 in gliomas and which nearly always occur in a heterozygous manner leaving one parental allele unaffected, strongly compromise the ability of the enzyme to decarboxylate isocitrate to $\alpha$-ketoglutarate and to generate NADPH $[9,26]$. In contrast, mutated IDH1 protein gains a novel function enabling the conversion of $\alpha$-ketoglutarate to 2-hydroxyglutarate in a $\mathrm{NADPH}$-consuming manner [5, 21]. While 2-hydroxyglutarate appears to increase the levels of reactive oxygen species, its role for tumor development is not clear. The mutation causes reduced catalytic generation of $\alpha$-ketoglutarate which combined with its additional consumption due to the gained function may inhibit prolyl hydroxylases thereby resulting in activation of the transcription factor, hypoxia-inducible factor (HIF) [27].

The objective of our present study was to investigate whether the IDH1 mutation status provides an essential contribution to delineate the individual prognosis in patients with anaplastic astrocytoma or primary glioblastoma, respectively, and to assess whether the WHO grading system should be amended in this regard. For this purpose, we assembled a large cohort of anaplastic astrocytoma and glioblastoma patients who were treated and prospectively followed up within the NOA-04 trial or the German Glioma Network. The prognostic impact of IDH1 mutation and MGMT promoter methylation in these patients was assessed in relation to the histological classification. Based on our results, we propose a refinement of the current $\mathrm{WHO}$ classification of high-grade astrocytic gliomas that considers the IDH1 mutation status in addition to the classic histological parameters. 


\section{Material and Methods}

\section{Patients, clinical and molecular data and immunohistochemistry}

A group of 382 patients with anaplastic astrocytoma $(n=145)$ or primary glioblastoma $(n=237)$ forms the basis of this study. Clinical and molecular data are derived from 94 patients with anaplastic astrocytoma treated in the NOA-04 trial [25] and from 51 patients with anaplastic astrocytoma and 237 patients with glioblastoma included in the prospective translational cohort study of the German Glioma Network (GGN) [23]. None of the glioblastoma patients in this study had a history of previous manifestation as diffuse astrocytoma WHO grade II or anaplastic astrocytoma and all cases from both studies were centrally reviewed by the same neuropathologist (T.P.) at the German Brain Tumor Reference Center of the German Society for Neuropathology and Neuroanatomy (DGNN) according to the revised WHO 2000 and 2007 classifications [11], assuring identical histological classification and grading. Data from both studies were compiled in a joint database and centrally analyzed. Data from the NOA-04 trial [25] and data from the ongoing translational study of the GGN [23] have been published previously.

The methods and conditions for determining the mutational status of IDH1 by direct sequencing of PCR products has been described previously [23, 25]. Methods and conditions for detecting the IDH1R132H mutation by IHC with mouse monoclonal antibody H09 (Dianova, catalog number DIA H09, Hamburg, Germany) on an automated immunostainer (BenchMark, Ventana Medical Systems, Tuscon, AZ, USA) have been described in detail elsewhere [3-4]. Analysis for MGMT promoter methylation by methylation-specific PCR (MSP) was performed as previously described $[23,25]$.

Patients in this study were recruited between January 2004 and August 2008.-The GGN data were used with a cut-off value for last information of December $31^{\text {st }} 2009$. The extent of resection was assessed by early ( $<72$ hours) magnetic resonance imaging (MRI) or computed tomography (CT) but was not centrally reviewed. Complete resection was scored whenever no residual tumor was detected; subtotal resection corresponded to removal of $50 \%$ to $99 \%$ of the tumor and partial resection to removal of less than $50 \%$ of the tumor. Overall survival (OS) was the primary endpoint of most analyses which is most appropriate for this retrospective analysis of pooled patients from a clinical trial (NOA-04) and a large cohort study (GGN). Data on postsurgical first-line treatment and treatment at 
first recurrence of the patients were collected. The median observation time for the entire cohort was 41 months.

\section{Statistics}

The association of clinical data and molecular markers were tested by $\mathrm{x} 2$-test and Fisher's exact test. Predictive values, sensitivity and specificity with $95 \%-\mathrm{Cl}$ were determined to assess the validity of IHC to IDH1 mutations in comparison to DNA sequencing as gold standard. OS, the primary endpoint, was calculated from the day of first surgery until death or end of follow up. Logrank test was used to compare outcome data. Cox regression models were fitted to assess the independent impact of the $I D H 1$ mutation (mutated vs. wild type), adjusting for age (>60 vs. $\leq 60$ ), diagnosis (anaplastic astrocytoma vs. glioblastoma), MGMT-status (methylated yes vs. not methylated), and extent of resection (total vs. not total). Interaction between IDH1 mutation and histological diagnosis as well MGMT promoter methylation and histological diagnosis were evaluated in multivariate models. Data were analyzed by PASW Statistics 18 (Version 18.0.0) and StatXact-8 (Cytel Studio Version 8.0.0). 


\section{Results}

\section{Patient characteristics}

Patient characteristics are given in table 1. As expected, patients with anaplastic astrocytoma were 17 years younger (median) than patients with glioblastoma, had better performance status, experienced somewhat less radical surgery and less frequently received combined modality treatment as first-line treatment. Hence any analysis of prognostic factors needed adjustment for these imbalances in order to separate the contribution of each single factor.

\section{IDH1 sequencing and immunohistochemical data}

IDH1 mutations were detected in 104 of 382 high grade malignant astrocytic gliomas. In 145 anaplastic astrocytomas we found 87 mutations $(60.0 \%)$ with 78 of the R132H, 6 of the R132C, 2 of the R132S and 1 of the R132G types. In 237 glioblastomas we found 17 IDH1 mutations (7.2\%) which were all of the R132H type. All mutations in anaplastic astrocytomas and glioblastomas were heterozygous. IDH2 mutations were analyzed in 365 patients of the 382 patients. Only a single glioblastoma patient carried an IDH2 R172K mutation.

A total of 197 cases consisting of 80 anaplastic astrocytomas and 117 glioblastomas were analyzed by IHC with monoclonal antibody $\mathrm{H} 09$ specific for mutant IDH1 protein encoded by the IDH1R132H mutation. We detected mutant IDH1 protein in 40 anaplastic astrocytomas and in 19 glioblastomas. Seven mutations which were recognized by sequencing were not detected by the mutation-specific antibody. These included five patients with four R132C and one R132S mutations which were not recognized by the mutation-specific antibody and two patients which were sequenced with the IDH1R132H mutation but did not exhibit antibody binding. On the other hand three cases were detected with the antibody but had escaped initial sequencing due to a signal below threshold. However, re-sequencing confirmed the mutation. Thus all IHC-positive cases were confirmed by sequencing.

Hence, the positive predictive value (PPV) of IHC for IDH1 R132H mutations is $100 \%(95 \%$ CI $93.4 \%$ to $100 \%$ ), while the negative predictive value (NPV) of a negative IHC for excluding IDH1mutations is $94.9 \%(95 \%-\mathrm{Cl} 89.8 \%$ to $97.4 \%)$. Sensitivity is $89.4 \%(95 \%-\mathrm{Cl} 79.4 \%$ to $95.6 \%)$ and specificity $100 \%(95 \%-\mathrm{Cl} 97.2 \%$ to $100 \%)$. This implies that IHC is highly predictive of the sequencing result and can therefore be used as a very powerful surrogate marker of the IDH1 sequence status. 


\section{MGMT promoter methylation}

The MGMT promoter methylation status was determined in 338 patients including 105 anaplastic astrocytoma and 233 glioblastoma patients. Among anaplastic astrocytoma patients 58 exhibited MGMT promoter methylation while 47 did not. Among glioblastomas, 110 exhibited a methylated MGMT promoter while 123 did not. MGMT and IDH1 status in the 105 anaplastic astrocytomas and 233 glioblastomas are given in table 1. This table also provides the relationships between molecular findings for both genes and patient age groups. It is noteworthy that MGMT promoter methylation was more frequent in $I D H 1$ mutated tumors $(69 \%, 53 / 77)$ than in wild type-IDH1-tumors $(44 \%, 115 / 261)$, irrespective of the histological diagnosis $(p=0.0001)$.

\section{Survival (univariate analysis)}

Figure 1 shows OS estimates in a univariate breakdown regarding the main factors diagnosis, age, IDH1 status and MGMT status. Patients with anaplastic astrocytoma lived significantly longer than patients with glioblastoma $(\mathrm{p}<0.0001)$ (figure $1 \mathrm{a})$. The median OS was 42.5 months $(95 \%-\mathrm{Cl} 31.9$ to 53.2) for anaplastic astrocytoma and 13.6 months $(95 \%-\mathrm{Cl} 11.6$ to 15.6$)$ for glioblastoma. Patients with IDH1 mutations lived longer than patients without $(\mathrm{p}<0.0001)$ (figure $1 \mathrm{~b})$, Patients with $M G M T$ promoter methylation lived longer than patients without $(p<0.0001)$ (figure $1 c)$ and furthermore, we confirmed younger age at diagnosis as a significant prognostic factor associated with longer OS $(p<0.0001)$ (figure 1d). When we calculated the relative risks $(R R)$ for these pair-wise comparisons, we found the largest risk for IDH1 wild type versus mutant (RR=5.1; $95 \%-\mathrm{Cl} 3.5$ to 7.5$)$ followed by glioblastoma versus anaplastic astrocytoma $(\mathrm{RR}=3.4 ; 95 \%-\mathrm{Cl} 2.5$ to 4.6$)$, old versus young age (RR=3.3; 95\%-Cl 2.6 to 4.3 ) and MGMT non-methylated versus methylated (RR=2.3;95\%-Cl 1.7 to 3). These findings are already indicative of a strong prognostic contribution of the IDH1 mutation status. However, these univariate contrasts are confounded by the unbalanced compositions of the groups. Hence, multivariate modeling is required to adjust for these effects.

\section{Survival (multivariate analysis)}

Figures 2-4 provide insights into the multivariate structure of the survival data. For this purpose, we separated the OS estimates in subgroups using two (or three) main factors. Co-evaluation of 
diagnosis and MGMT status showed that both factors contribute to prognosis. MGMT splits the cohort into the most favorable clinical course for 47 anaplastic astrocytoma patients with MGMT promoter methylation, followed by 58 anaplastic astrocytoma patients without MGMT promoter methylation, followed by 110 glioblastoma patients with and trailed by 123 glioblastoma patients without MGMT promoter methylation (figure 2a). There is also an indication that the prognostic split associated with MGMT is slightly more pronounced in patients with glioblastoma compared to patients with anaplastic astrocytoma (see below).

Co-evaluation of diagnosis and age indicates that both factors contribute to prognosis. Anaplastic astrocytoma patients aged 60 years or younger had the most favorable clinical course. The poorest survival occurred in glioblastoma patients older than 60 years. The age-effect seems to be more pronounced among anaplastic astrocytoma patients (figure $2 \mathrm{~b}$ ).

Co-evaluation of histological diagnosis and IDH1 status showed that both factors contribute to prognosis. The most favorable OS was found in 87 anaplastic astrocytoma patients with IDH1 mutation, followed by 17 glioblastoma patients with $I D H 1$ mutation $(p=0.014)$. The difference between the 17 glioblastoma patients with IDH1 mutation and 58 anaplastic astrocytoma patients without IDH1 mutation was not significant $(p=0.222)$. However, the Kaplan-Meier plots indicate a trend towards an inferior OS for patients with anaplastic astrocytoma patients without IDH1 mutation. These patients showed a longer OS than 220 glioblastoma patients without $I D H 1$ mutation $(p=0.003)$. The differences in OS across the whole cohort were highly significant $(p<0.0001)$ (figure $3 a)$. The separation of these four groups became even more prominent when PFS was analyzed instead of OS (figure $3 b$ ), but, as indicated above, PFS may not be an appropriate endpoint for a pooled analysis of a clinical trial where single modality treatment was the rule (NOA-04) and a cohort where single modality treatment was more likely given to patients with a more malignant tumor with less favorable prognostic factors (GGN).

An analysis restricted to patients aged 60 years or younger again showed best OS in 78 anaplastic astrocytomas with IDH1 mutation. Worst outcome was seen in 96 glioblastoma patients without IDH1 mutation ( $p<0.0001$ ) (figure 3c). If we restricted the analysis to patients older than 60 years, we find $I D H 1$ mutations only rarely ( $\mathrm{N}=2$ for glioblastoma, $\mathrm{N}=9$ for anaplastic astrocytoma) and these curves are not shown. However, we found that there was no apparent prognostic difference for anaplastic astrocytoma and glioblastoma patients without IDH1 mutation $(p=0.457)$ (figure $3 \mathrm{~d})$. 
Hence, elderly anaplastic astrocytoma patients without IDH1 mutation seem to have the same poor prognosis as glioblastoma patients.

Co-evaluation of IDH1 and MGMT status revealed that MGMT permits to separate the prognostic difference by IDH1 mutations further. However, patients with IDH1 mutation performed better than patients without IDH1 mutation irrespective of MGMT status. Patients without IDH1 mutation but with MGMT promoter methylation fared worse and poorest survival was seen in patients showing neither IDH1 mutation nor MGMT promoter methylation (figure 4).

Figure 5 shows the co-evaluation of the three main factors diagnosis, IDH1 and MGMT status. Clearly the dominant prognostic factors are related to the molecular status. There is a very poor prognostic group without IDH1 mutation and with unmethylated MGMT at one end of the spectrum (with few long-term survivors) and a small but very favorable group of IDH1 mutant and MGMT methylated patients (among whom long-term survival seems frequent). In most of the four groups, WHO diagnosis seemed to retain a small influence on OS. Among patients without IDH1 mutation and without MGMT promoter methylation, the 28 patients with anaplastic astrocytoma fared better $(p=0.0012)$ than the 118 with glioblastoma (figure $5 a)$. There was no significant difference $(p=0.1824)$ in survival between 17 anaplastic astrocytoma and 98 glioblastoma patients without IDH1 mutation and with MGMT promoter methylation (figure 5b). Similarly, 41 anaplastic astrocytoma patients with IDH1 mutation and with MGMT promoter methylation did not live significantly longer $(p=0.3534)$ than 12 glioblastoma patients with the same molecular findings (figure $5 \mathrm{~d}$ ). The group defined by patients with IDH1 mutation and without MGMT promoter methylation included only 19 anaplastic astrocytoma and 5 glioblastoma patients and, therefore, was too small to yield conclusive results (figure $5 c$ ).

\section{Multivariate modeling}

Multivariate modeling was undertaken to estimate the RR for age, extent of resection, diagnosis, IDH1 mutation status and MGMT status. The results for the main effects are given in table 2. IDH1 mutation was the dominant prognostic factor with a RR of 2.7 (95\% Cl 1.6 to 4.5). Histology and MGMT status were also prominent with RR of 2.2 respectively. Since RR can be multiplied, it becomes evident that this implies a very powerful system spanning a RR range of about 13 in total $(2.7 \times 2.2 \times 2.2)$. Finally, in a sensitivity analysis, we also introduced two interaction terms into the main effects model. The model fit (i.e. log-likelihood) improved significantly. This model provides an indication that the $I D H 1$ effect is quantitatively more pronounced in anaplastic astrocytoma (anaplastic astrocytoma: 
$\mathrm{RR}=4.1$ glioblastoma: $\mathrm{RR}=1.8$ ) while the $M G M T$ effect is more pronounced in glioblastoma (anaplastic astrocytoma: $\mathrm{RR}=1.3$; glioblastoma: $\mathrm{RR}=2.5)$. 


\section{Discussion}

Classification and grading of high-grade astrocytic gliomas according to WHO is accompanied by a considerable interobserver variation [19]. Reasons are guidelines allowing for subjective interpretation. On the one hand, this may be due to a subjective interpretation of borderline histological features by different neuropathologists. On the other hand, malignant gliomas are regionally heterogeneous tumors and incomplete tissue sampling thus may lead to the underestimation of a tumor's true malignancy grade when important histological features of anaplasia are restricted to focal areas and not represented in the evaluable tissue specimens. Molecular analyses are expected to reduce diagnostic interobserver variation due to providing clear yes/no answers in many instances and detecting molecular changes present in all tumor cells, thereby reducing the problems of subjectivity in histological assessment and incomplete tissue sampling. In the present study we focus on the role of the IDH1 status in assisting classification and grading of anaplastic astrocytomas and glioblastomas. The data set analyzed here was obtained by joining the primary data sets of the anaplastic astrocytoma patients treated within the NOA-04 randomized phase III trial [25], the glioblastoma cohort of the GGN [23], and a new subset of anaplastic astrocytomas from the GGN. In our series $87 / 145(60 \%)$ anaplastic astrocytomas and 17/137 (7.2\%) glioblastomas carried a mutation in IDH1. The frequency of $I D H 1$ mutations in anaplastic astrocytomas in our series is within the spectrum described in previous series ranging from $52 \%$ [9] to $78 \%$ [22]. The frequency of $I D H 1$ mutation detected in glioblastomas has generally been reported to range below $10 \%$ in primary glioblastoma [1, $9,22,26]$.

The impact of $I D H 1$ mutations on clinical outcome has been demonstrated in prospective clinical studies including anaplastic astrocytomas and glioblastomas [20, 23, 25]. It has also been detected in various retrospective series including diffuse gliomas of WHO grades II, III and IV recently reviewed [15]. There is consensus on patients with IDH1 mutations performing better than those without $[6,9,14,17,26]$. Our data provide compelling evidence that the IDH1 status separates anaplastic astrocytomas and glioblastomas in two sub-entities each with significantly different clinical outcomes. Further, our data indicate a close clinical and biological relation of anaplastic astrocytomas and glioblastomas with IDH1 mutation versus anaplastic astrocytomas and glioblastomas without IDH1 mutations. The sequence from more favorable to poorer outcome was 1) IDH1 mutant anaplastic astrocytoma, 2) IDH1 mutant glioblastoma, 3) IDH1 wild type anaplastic astrocytoma and 4) IDH1 wild 
type glioblastoma, respectively. The missing significance between groups 2 and $3(p=0.222)$ might be due to the low number of patients with IDH1 mutant glioblastoma $(n=17)$. The prognostic relevance of IDH1 status in comparison with histopathological evaluation of high-grade astrocytomas is demonstrated in figures $1 \mathrm{a}$ and $\mathrm{b}$ and figure 5 and particularly in the multivariate modeling result Using the IDH1 mutation status as a separator to this predefined set of tumors, we were able to separate two patient groups with even more pronounced differences in median OS than those stratified according to conventional histological features. Thus, IDH1 analysis in this set of tumors is a more powerful prognostic marker than current WHO classification and grading for high-grade astrocytomas. Notably, IDH1 wild type anaplastic astrocytoma patients demonstrated not only shorter OS when compared to IDH1 mutant anaplastic astrocytoma patients but even survived shorter than patients with IDH1 mutant glioblastoma.

Apart from the WHO diagnosis, patient age has been established as a powerful parameter for the prognosis of patients with anaplastic astrocytomas and glioblastomas [2]. Therefore, we also examined the relationship between $I D H 1$ status and patient age. Analysis in patients aged 60 years or younger or over 60 revealed that in the younger age group, anaplastic astrocytoma patients without $I D H 1$ mutations exhibited OS very similar to that of glioblastoma patients with IDH1 mutations (figure 3c). Importantly, in patients over 60 years the absence of IDH1 mutation in anaplastic astrocytoma was indicative of poor OS that was similar to the OS of older patients with IDH1 wild type glioblastoma (Figure 3c). This may be of relevance for treatment decisions.

A yet unsolved issue relates to the impact of $I D H 2$ mutation on OS. So far, none of the previous series provided reliable data on the clinical relevance of IDH2 mutations alone [20]. IDH2 mutations occur infrequently in astrocytic gliomas. In a large series including astrocytomas, oligodendrogliomas and oligoastrocytomas, the frequency of $I D H 2$ mutations was $3.1 \%$. Of note, IDH2 mutations clustered in patients with oligodendroglial tumors but were found in less than $1 \%$ of astrocytomas [7]. We examined 132 anaplastic astrocytoma patients and 233 glioblastoma patients from the present series for $I D H 2$ mutation in codon 172 and detected only a single R172K mutation in a glioblastoma patient. Because $I D H 2$ mutations are so rare in anaplastic astrocytomas and glioblastomas, this molecular alteration was not further considered in the clinical correlations performed in the present study.

MGMT promoter methylation status has been demonstrated as a powerful prognostic and predictive marker for patients with glioblastomas $[8,24]$. Its relevance was confirmed in our series to 
be independent of the IDH1 status (figure 2a, 5). For all four groups, stratified for diagnosis and presence or absence of $I D H 1$ mutation, OS was longer in patients with MGMT promoter methylation. These differences were significant for glioblastomas with or without IDH1 mutation, and for anaplastic astrocytomas with IDH1 mutation. In contrast, the MGMT status was not associated with differential survival in anaplastic astrocytomas without IDH1 mutation. Interestingly, the rate of patients with MGMT methylation was higher in both anaplastic astrocytomas and glioblastomas with IDH1 mutation than in anaplastic astrocytomas and glioblastomas without IDH1 mutation. Upon sorting our series of 338 patients into four groups defined by combined IDH1 and MGMT status, it became evident that the histological distinction between anaplastic astrocytomas and glioblastomas had only a moderate effect on OS (figures $5 a-5 d$ ). Noteworthy is the very similar gradient of the curves for both histological diagnoses within each group. This analysis underscores the importance of combined IDH1 and MGMT analysis to predict survival in these patients.

We recently developed a monoclonal mouse antibody $(\mathrm{m} / \mathrm{DH} 1 \mathrm{R} 132 \mathrm{H} /$ clone $\mathrm{H} 09)$ to mutant IDH1 protein of the $\mathrm{R} 132 \mathrm{H}$ type [3-4]. The $\mathrm{R} 132 \mathrm{H}$ mutation constitutes more than $90 \%$ of the IDH1 mutations seen in gliomas [7]. Therefore, we performed immunohistochemistry with this antibody on 197 tumors of our series, which revealed high positive and negative predictive values of $\mathrm{H} 09 \mathrm{IHC}$ for detecting IDH1 mutation, thus corroborating this method as a surrogate approach to DNA sequencing. Representative data for IHC with mouse monoclonal antibody H09 is shown in figure 6. Interestingly, we detected in three samples mutant IDH1 protein of the $\mathrm{R} 132 \mathrm{H}$ type that showed no IDH1 mutation by initial sequencing. However, these IDH1 mutations were confirmed by re-sequencing. On the other hand, two IDH1 R132H mutations were not detected by the mIDH1R132H/clone $\mathrm{H} 09$ antibody. These findings show that both methods do not have a $100 \%$ detection rate but that detection rate is comparable.

Based on the presented data we propose to consider a refinement of the current WHO classification and grading system for anaplastic astrocytomas and glioblastomas by subdividing each tumor entity into two molecularly and clinically distinct subgroups according to the IDH1 mutation status. This implies that this molecular parameter will become part of the tumor classification and thus needs to be determined on a regular basis. In detail, IDH1 mutant anaplastic astrocytomas should be separated from IDH1 wild type anaplastic astrocytomas, and likewise, glioblastomas with and without IDH1 mutation should be distinguished. It remains to be seen whether further molecular subdivision of glioblastomas according to the MGMT promoter methylation status will be applicable in the routine 
diagnostic setting. In contrast to the technically simple and reliable detection of IDH1 mutations by DNA sequencing or immunohistochemistry, current methods for the molecular analysis of MGMT promoter methylation are technically more sophisticated, difficult to standardize, and thus suffer from considerable interlaboratory variability [15]. We expect an extended sub-classification of anaplastic astrocytomas and glioblastomas to be of immediate clinical impact. For example, our data clearly indicate that patients with IDH1 wild type anaplastic astrocytomas have poor clinical outcome. Thus, one could consider treating these patients more aggressively with first-line combined radiochemotherapy corresponding to the current standard of care for glioblastoma treatment. On the other hand, the rare IDH1 mutant primary glioblastomas may require less aggressive first-line treatment similar to that usually administered to anaplastic astrocytoma patients, that is, either radiotherapy or alkylating chemotherapy, but no combined modality treatment [25]. As IDH1 mutant glioblastomas usually affect younger patients and are associated with a higher likelihood of long-term survival, a less aggressive up-front treatment may be beneficial also in terms of reducing treatmentassociated neurotoxicity in these patients. It is not yet clear whether anaplastic astrocytomas without IDH1 mutation generally should be considered as underdiagnosed glioblastomas. While such an approach would yield a tighter correlation of diagnosis and OS than the current procedure, it does not account for the still better OS of anaplastic astrocytoma patients without IDH1 mutation than glioblastoma patients without IDH1 mutations. We also assume that our data will have major impact on reporting results from clinical trials and on the study designs for novel trials focusing on anaplastic astrocytomas and glioblastomas. The circumstance that the presence of IDH1 mutations is the strongest indicator for more favorable clinical outcome will make it inevitable to determine this parameter in order to test the influence of the experimental treatment applied. Furthermore, this molecular parameter likely will have an impact on the inclusion criteria of future trials.

How could the WHO classification be developed in the future with regard to the optimal prognostic and clinically most helpful stratification of patients with high-grade astrocytic gliomas? There are several possibilities for this separation, such as the simple addition of the IDH1 status to the histological diagnosis to indicate that the respective tumor is associated with a more or less favorable clinical course. A more radical approach would be to disregard necrosis and microvascular proliferation as the basis for distinction between anaplastic astrocytomas and glioblastomas, and substitute these histological parameters by the IDH1 mutation status, that is high-grade astrocytic tumors with IDH1 mutation would be termed anaplastic astrocytomas while all high-grade astrocytic 
tumors without IDH1 mutation would be termed glioblastomas. Such a procedure would result in a significantly tighter association of grading with clinical course and, therefore, would better suit the intention of glioma grading to provide a prognostically meaningful stratification that may guide the postoperative treatment. It would emphasize on biological properties inherent of distinct tumor cell lineages, i.e., astrocytoma deriving from a tumor precursor cell characterized by IDH1 mutation. However, it would also break with a long and well received tradition of purely histological grading by devaluating the prognostic importance of necrosis or microvascular proliferation. In fact, this approach would miss some prognostically relevant information, as indicated by the survival differences between anaplastic astrocytomas and glioblastomas within the group of $I D H 1$ wild type tumors. IDH1 mutation is frequent in secondary glioblastomas derived by progression from astrocytomas. It has been suggested that IDH1 mutant primary glioblastoma in fact represent secondary glioblastoma that rapidly evolved from lower grade precursor tumors and thus have not been diagnosed before [13]. Thus, secondary glioblastomas and IDH1 mutant primary glioblastomas could be the endpoints of a disease following the same pathogenetic pathway. This hypothesis is supported by similar chromosomal and genetic aberration profiles in these two types of glioblastomas $[13,18]$ and might be taken into account by terming all IDH1 mutant glioblastomas secondary glioblastomas, thereby distinguishing them from the more common primary glioblastomas that are IDH1 wild type. However, there undoubtedly is a fraction of true secondary glioblastomas that progressed from a lower grade astrocytoma but lack IDH1 mutation, consistent with the lack of IDH1 mutations in approximately $20 \%$ of lower grade astrocytomas $[1,9,22,26]$. These tumors argue against redefining the clinically established term of secondary glioblastoma. Another alternative might be to use the traditional designation "anaplastic astrocytoma WHO grade III" only for those anaplastic astrocytomas that carry an IDH1 mutation, while the term "anaplastic astrocytoma WHO grade IV" may be used for IDH1 wild type anaplastic astrocytomas. However, this designation is problematic as glioblastoma is traditionally regarded as the only type of astrocytic glioma corresponding to WHO grade IV. Furthermore, the WHO grade IV group would then cover a spectrum of malignant gliomas variably defined on histological and/or molecular features, and associated with different prognoses, i.e., IDH1 mutant and wild type glioblastomas, glioblastomas with oligodendroglial component or "anaplastic oligoastrocytoma WHO grade IV" [16], which show an intermediate prognosis between anaplastic oligoastrocytoma and glioblastoma [12], and IDH1 wild type anaplastic astrocytoma. Thus, for the time being and for reasons of simplicity, we propose to continue using the conventional histological 
terms of anaplastic astrocytoma and glioblastoma, respectively, supplemented by the IDH1 mutation status, and alert the physicians treating the patients concerning the prognostic implications of this molecular marker.

In conclusion, we demonstrate that the IDH1 mutation status distinguishes anaplastic astrocytomas and glioblastomas into clinically meaningful prognostically distinct subgroups that possibly require different first-line treatment. Therefore, determination of $I D H 1$ status is essential for a comprehensive neuropathological assessment of high-grade astrocytic gliomas and should be considered in the design and evaluation of future clinical trials. The differential distribution of IDH1 mutations across age groups largely explains the prognostic impact of age in high-grade astrocytoma patients. We propose to revise the current WHO classification of these tumors by inclusion of the IDH1 mutation status, which can be easily and reliably assessed by DNA sequencing or IHC. 


\section{Acknowledgements}

This work was supported by the German Cancer Aid (Deutsche Krebshilfe Deutsche Krebshilfe 703163-Wi 3) and the Bundesministerium für Bildung und Forschung (BMBF - 01ES0729, 01ES0730 and 01GS0883). Writing committee: A. von Deimling, M. Loeffler, M. Weller, B. Hentschel, C. Hartmann. Further contributors to this publication were (a) for lab work K. Lindenberg (University Heidelberg), B. Wagner (University Bonn), K. Kaulich (University Düsseldorf), (b) for data base and data management U. Schoenwiese, R. Stein, J. Gietzelt (University Leipzig), (c) for patient enrolment M. Tatagiba, B. Braun, A. Bächle (University Tübingen), S. Ott, B. Harzheim (University Bonn), O. Heese, M. Beyer, S. Winkler (University Hamburg), D. Krex, A. Sorokin (University Dresden), G. Nikkah, T. Reithmeier, C. Weis (University Freiburg), J.C. Tonn, O. Schnell, M. Deschner (University München), M. Weller was the PI, W. Wick the coordinator of the NOA-04 trial; various centers in Germany contributed to patient enrolment in this trial.

We are greatly indebted to all participants of the German Glioma Network and the German NeuroOncology Group for their invaluable contributions. 


\section{References}

1. Balss J, Meyer J, Mueller W, et al. (2008) Analysis of the IDH1 codon 132 mutation in brain tumors. Acta Neuropathologica 116: 597-602

2. Burger PC, Vogel FS, Green SB, et al. (1985) Glioblastoma multiforme and anaplastic astrocytoma, pathologic criteria and prognostic implications. Cancer 56: 1106-1111

3. Capper D, Weißert S, Balss J, et al. (2010) Characterization of R132H Mutation Specific IDH1 Antibody binding in brain tumors. Brain Pathology 20: 245-254

4. Capper D, Zentgraf H, Balss J, et al. (2009) Monoclonal Antibody Specific for IDH1 R132H Mutation. Acta Neuropathologica Berlin 118: 599-601

5. Dang L, White DW, Gross S, et al. (2009) Cancer-associated IDH1 mutations produce 2hydroxyglutarate. Nature 462: 739-744

6. Gravendeel LA, Kloosterhof NK, Bralten LB, et al. (2010) Segregation of non-p.R132H mutations in IDH1 in distinct molecular subtypes of glioma. Hum Mutat 31: E1186-99

7. Hartmann C, Meyer J, Balss J, et al. (2009) Type and frequency of IDH1 and IDH2 mutations are related to astrocytic and oligodendroglial differentiation and age: A study of 1010 diffuse gliomas. Acta Neuropathologica 118: 469-474

8. Hegi ME, Diserens AC, Gorlia T, et al. (2005) MGMT gene silencing and benefit from temozolomide in glioblastoma. N Engl J Med 352: 997-1003

9. Ichimura K, Pearson DM, Kocialkowski S, et al. (2009) IDH1 mutations are present in the majority of common adult gliomas but are rare in primary glioblastomas. Neuro Oncol 11: 341 347

10. Kraus JA, Koopmann J, Kaskel P, et al. (1995) Shared allelic losses on chromosomes 1p and $19 q$ suggest a common origin of oligodendroglioma and oligoastrocytoma. Journal of Neuropathology and Experimental Neurology 54: 91-95

11. Louis D, Ohgaki H, Wiestler O, et al., eds. World Health Organization Classification of Tumours of the Central Nervous System. 4 ed. World Health Organization Classification of Tumours, ed. Bosman F, Jaffe E, Lakhani S, et al. 2007, IARC: Lyon. 
12. Miller CR, Dunham CP, Scheithauer BW, et al. (2006) Significance of necrosis in grading of oligodendroglial neoplasms: a clinicopathologic and genetic study of newly diagnosed highgrade gliomas. J Clin Oncol 24: 5419-26

13. Nobusawa $\mathrm{S}$, Watanabe $\mathrm{T}$, Kleihues $\mathrm{P}$, et al. (2009) IDH1 mutations as molecular signature and predictive factor of secondary glioblastomas. Clin Cancer Res 15: 6002-7

14. Parsons DW, Jones S, Zhang X, et al. (2008) An integrated genomic analysis of human glioblastoma multiforme. Science 321: 1807-12

15. Riemenschneider MJ, Jeuken JW, Wesseling P, et al. (2010) Molecular diagnostics of gliomas: state of the art. Acta Neuropathologica: epub ahead of print

16. Scheithauer BW, Fuller GN, VandenBerg SR (2008) The 2007 WHO classification of tumors of the nervous system: controversies in surgical neuropathology. Brain Pathol 18: 307-16

17. Sonoda Y, Kumabe T, Nakamura T, et al. (2009) Analysis of IDH1 and IDH2 mutations in Japanese glioma patients. Cancer Sci 100: 1996-8

18. Toedt G, Barbus S, Wolter M, et al. (2010t) Molecular Signatures Classify Astrocytic Gliomas by IDH1 Mutation Status. Int J Cancer : epub ahead of print

19. van den Bent MJ (2010) Interobserver variation of the histopathological diagnosis in clinical trials on glioma: a clinician's perspective. Acta Neuropathologica 120: 297-304

20. van den Bent MJ, Dubbink HJ, Marie Y, et al. (2010) IDH1 and IDH2 mutations are prognostic but not predictive for outcome in anaplastic oligodendroglial tumors: a report of the European Organization for Research and Treatment of Cancer Brain Tumor Group. Clin Cancer Res 16: $1597-604$

21. Ward PS, Patel J, Wise DR, et al. (2010) The Common Feature of Leukemia-Associated IDH1 and IDH2 Mutations Is a Neomorphic Enzyme Activity Converting alpha-Ketoglutarate to 2Hydroxyglutarate. Cancer Cell

22. Watanabe T, Nobusawa S, Kleihues P, et al. (2009) IDH1 Mutations Are Early Events in the Development of Astrocytomas and Oligodendrogliomas. American Journal of Pathology 174: 653-656

23. Weller M, Felsberg J, Hartmann C, et al. (2009) Molecular predictors of progression-free and overall survival in patients with newly diagnosed glioblastoma: a prospective translational study of the German Glioma Network. Journal of Clinical Oncology 27: 5743-5750 
24. Weller M, Stupp R, Reifenberger G, et al. (2010) MGMT promoter methylation in malignant gliomas: ready for personalized medicine? Nat Rev Neurol 6: 39-51

25. Wick W, Hartmann C, Engel C, et al. (2009) NOA-04 randomized phase III trial of sequential radiochemotherapy of anaplastic glioma with procarbazine, lomustine, and vincristine or temozolomide. Journal of Clinical Oncology 27: 5874-5880

26. Yan H, Parsons DW, Jin G, et al. (2009) IDH1 and IDH2 Mutations in Gliomas. N Engl J Med 360: $765-773$

27. Zhao S, Lin Y, Xu W, et al. (2009) Glioma-derived mutations in IDH1 dominantly inhibit IDH1 catalytic activity and induce HIF-1alpha. Science $324: 261-5$ 
table 1

Patient characteristics

\begin{tabular}{|c|c|c|}
\hline & $\begin{array}{c}\text { A III } \\
\mathrm{N}=145(100 \%)\end{array}$ & $\begin{array}{c}\text { GBM } \\
\mathrm{N}=237(100 \%)\end{array}$ \\
\hline Age in years (median, range) & $\begin{array}{c}43.7 \\
(18.3-77.8)\end{array}$ & $\begin{array}{c}60.9 \\
(19.2-86.5)\end{array}$ \\
\hline Gender (male/female) & $89 / 56$ & $144 / 93$ \\
\hline \multicolumn{3}{|l|}{ Extent of resection $\mathbf{n}(\%)$} \\
\hline Complete & $48(33.1 \%)$ & $112(47.3 \%)$ \\
\hline Subtotal (50-99\%) & $53(36.6 \%)$ & $81(34.2 \%)$ \\
\hline Partial $(<50 \%)$ & $43(29.7 \%)$ & $23(9.7 \%)$ \\
\hline Biopsy & $1(0.7 \%)$ & $21(8.9 \%)$ \\
\hline $\operatorname{KPS}(\leq 70 />70)$ & $10 / 127$ & $51 / 181$ \\
\hline \multicolumn{3}{|l|}{ First-line treatment } \\
\hline RT alone & $63(43.4 \%)$ & $60(25.3 \%)$ \\
\hline RT and CT (alkylating agents) & $29(20.0 \%)$ & $164(69.2 \%)$ \\
\hline CT alone (alkylating agents) & $49(33.8 \%)$ & $10(4.2 \%)$ \\
\hline None & $4(2.8 \%)$ & $3(1.3 \%)$ \\
\hline \multicolumn{3}{|l|}{ Treatment at first recurrence } \\
\hline RT alone & $42(29.0 \%)$ & $2(0.8 \%)$ \\
\hline RT and CT (alkylating agents) & $1(0.7 \%)$ & $15(6.3 \%)$ \\
\hline CT alone (alkylating agents) & $45(31.0 \%)$ & $54(22.8 \%)$ \\
\hline Other CT +/- RT & 0 & $12(5.1 \%)$ \\
\hline None & $57(39.3 \%)$ & $154(65.0 \%)$ \\
\hline IDH1 mutated & $87(60.0 \%)$ & $17(7.2 \%)$ \\
\hline$\leq 60$ years & $78(53.8 \%)$ & $15(6.2 \%)$ \\
\hline$>60$ years & $9(6.2 \%)$ & $2(1.0 \%)$ \\
\hline MGMT promoter methylated & $58 / 105(55.2 \%)$ & $110 / 233(47.2 \%)$ \\
\hline$\leq 60$ years & $50(47.6 \%)$ & $53(22.7 \%)$ \\
\hline$>60$ Years & $8(7.6 \%)$ & $57(24.5 \%)$ \\
\hline$I D H 1$ and $M G M T(\mathrm{~N}=338)$ & $N=105$ & $N=233$ \\
\hline IDH1 wt and MGMT meth - & $28(26.7 \%)$ & $118(50.6 \%)$ \\
\hline IDH1 wt and MGMT meth + & $17(16.2 \%)$ & $98(42.1 \%)$ \\
\hline IDH1 mut and MGMT meth - & $19(18.1 \%)$ & $5(2.1 \%)$ \\
\hline IDH1 mut and MGMT meth + & $41(39.0 \%)$ & $12(5.2 \%)$ \\
\hline
\end{tabular}

Abbreviations: $\mathrm{RT}=$ radiotherapy $; \mathrm{CT}=$ chemotherapy; wt = wild type; mut = mutated; meth $-=$ unmethylated; meth + = methylated; $\mathrm{A}$ III = anaplastic astrocytoma $\mathrm{WHO}$ grade III; GBM = glioblastoma WHO grade IV 
table 2

Multivariate models

\begin{tabular}{|c|c|c|c|c|c|c|c|}
\hline & \multicolumn{3}{|c|}{ Model with main effects only } & & \multicolumn{3}{|c|}{$\begin{array}{l}\text { Model: main effects and } \\
\text { interactions with diagnosis }\end{array}$} \\
\hline & Relative Risk & 95\%-Cl & p-value & & $\begin{array}{l}\text { Relative } \\
\text { Risk }\end{array}$ & $\begin{array}{l}95 \%- \\
\mathrm{Cl}\end{array}$ & $\begin{array}{c}\mathrm{p}- \\
\text { value }\end{array}$ \\
\hline $\begin{array}{l}\text { Age } \\
\quad \leq 60 \\
>60\end{array}$ & $\begin{array}{c}1 \\
2.2\end{array}$ & 1.6 to 2.8 & $<0.001$ & $\begin{array}{l}\text { Age } \\
\qquad<=60 \\
>60\end{array}$ & $\begin{array}{c}1 \\
2.2\end{array}$ & $\begin{array}{l}1.7 \text { to } \\
2.9\end{array}$ & $\begin{array}{c}< \\
0.001\end{array}$ \\
\hline $\begin{array}{l}\text { Resection } \\
\text { no total } \\
\text { total }\end{array}$ & $\begin{array}{c}1 \\
0.7\end{array}$ & 0.5 to 0.9 & 0.009 & $\begin{array}{l}\text { Resection } \\
\text { no total } \\
\text { total }\end{array}$ & $\begin{array}{c}1 \\
0.7\end{array}$ & $\begin{array}{c}0.5 \text { to } \\
0.9\end{array}$ & 0.011 \\
\hline $\begin{array}{l}\text { Diagnosis } \\
\text { A III } \\
\text { GBM }\end{array}$ & $\begin{array}{c}1 \\
2.2\end{array}$ & 1.5 to 3.2 & $<0.001$ & $\begin{array}{c}\text { Diagnosis } \\
\text { A III } \\
\text { GBM }\end{array}$ & $\begin{array}{c}1 \\
2.7\end{array}$ & $\begin{array}{c}1.1 \text { to } \\
6.5\end{array}$ & 0.024 \\
\hline $\begin{array}{l}\text { IDH1 } \\
\text { general } \\
\text { mut } \\
\text { wt }\end{array}$ & $\begin{array}{c}1 \\
2.7\end{array}$ & 1.6 to 4.5 & $<0.001$ & $\begin{array}{l}\text { IDH1 in A III } \\
\text { mut } \\
\text { wt } \\
\text { IDH1 in GBM } \\
\text { mut } \\
\text { wt }\end{array}$ & $\begin{array}{c}1 \\
4.1 \\
\end{array}$ & \multicolumn{2}{|c|}{$\begin{array}{l}\text { Test for } \\
\text { interaction: } \\
p=0.099\end{array}$} \\
\hline $\begin{array}{c}\text { MGMT } \\
\text { general } \\
\text { meth + } \\
\text { meth - }\end{array}$ & $\begin{array}{c}1 \\
2.2\end{array}$ & 1.7 to 2.9 & $<0.001$ & $\begin{array}{c}\text { MGMT in A III } \\
\text { meth + } \\
\text { meth - } \\
\text { MGMT in GBM } \\
\text { meth + } \\
\text { meth - }\end{array}$ & $\begin{array}{c}1 \\
1.3 \\
\end{array}$ & \multicolumn{2}{|c|}{$\begin{array}{l}\text { Test for } \\
\text { interaction: } \\
p=0.067\end{array}$} \\
\hline
\end{tabular}

$\mathrm{wt}=$ wild type; mut $=$ mutant meth $-=$ unmethylated $;$ meth $+=$ methylated; A III = anaplastic astrocytoma WHO grade III; GBM = glioblastoma WHO grade IV 


\section{figure 1}
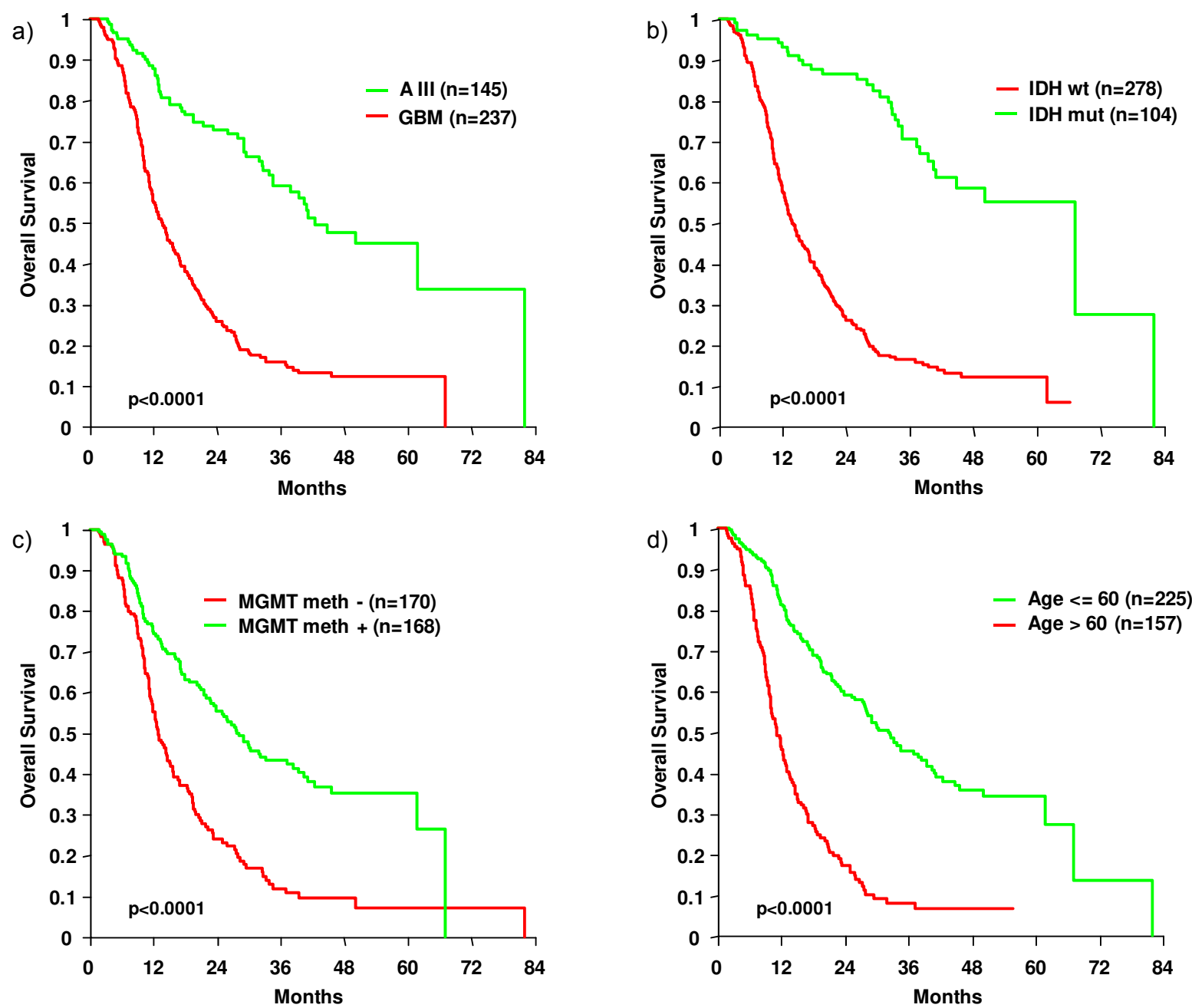

Kaplan-Meier plots of the series of 382 anaplastic astrocytomas and glioblastomas showing the association with overall survival of histological WHO diagnosis (a), IDH1 mutation status (b), MGMT methylation status (c) and age (d). 


\section{figure 2}
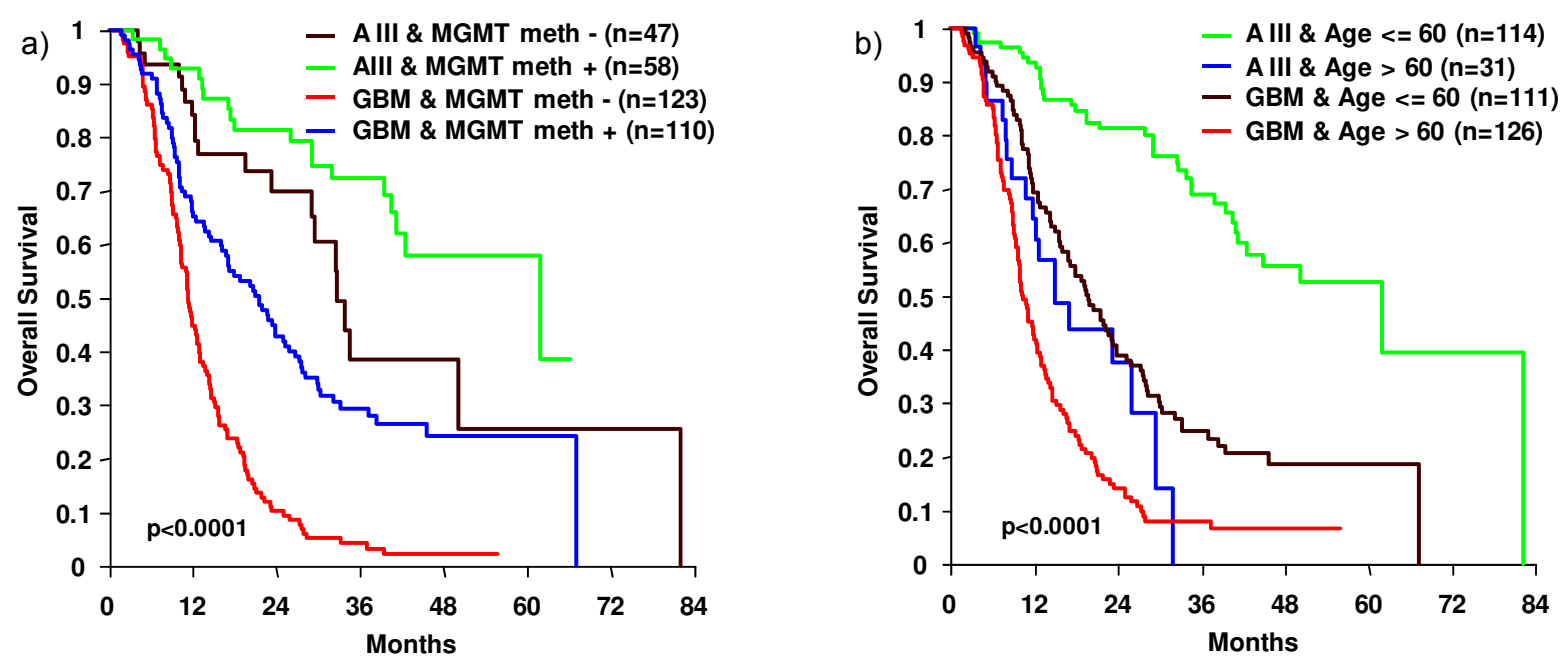

Kaplan-Meier plot of the series of 338 anaplastic astrocytomas and glioblastomas showing the association with overall survival of the combination of WHO diagnosis and MGMT methylation status (a). Association of overall survival with the effect of the combination of WHO diagnosis and age are shown on the series of 382 patients (b). 


\section{figure 3}
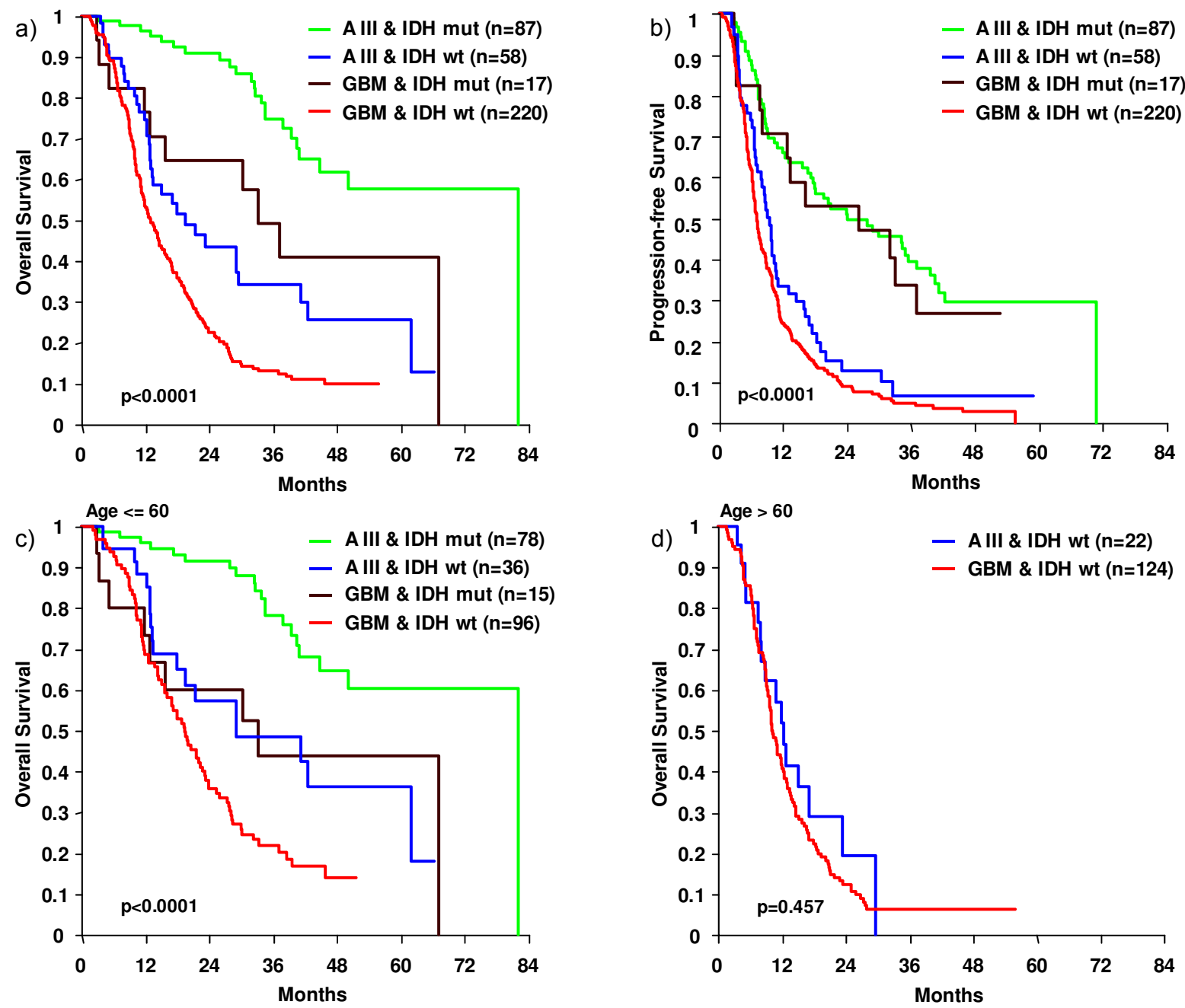

Kaplan-Meier plot of the series of 382 anaplastic astrocytomas and glioblastomas patients showing the association of $I D H 1$ status with overall survival (a) and progression-free survival (b). The same analysis regarding overall survival is shown for patients aged 60 years or younger (c). For patients older than 60 years, overall survival only for IDH1 wild type is shown (d). 


\section{figure 4}

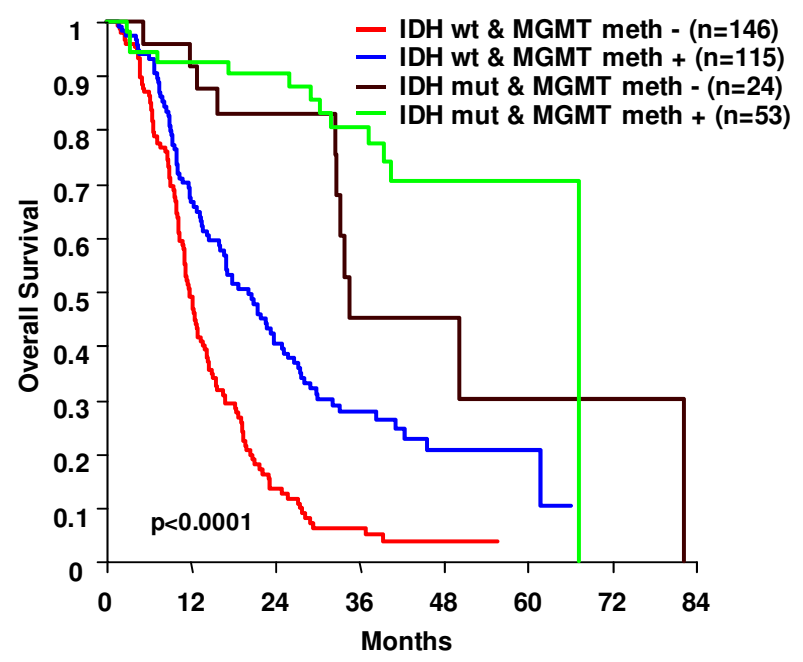

Kaplan-Meier plot of the series of 338 anaplastic astrocytomas and glioblastomas showing the combined associations of $I D H 1$ and MGMT status with overall survival. 


\section{figure 5}
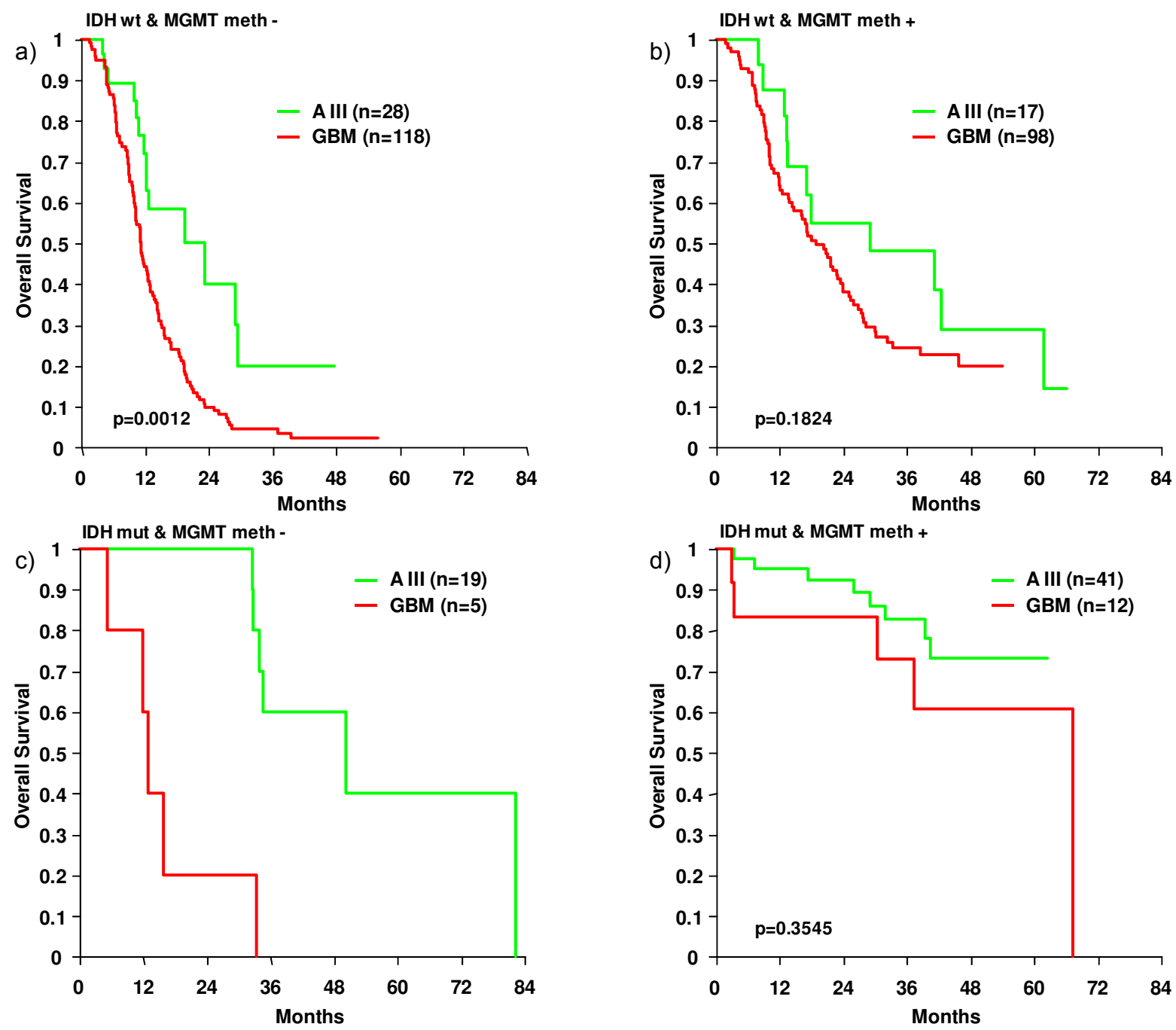

Kaplan-Meier plots of OS of anaplastic astrocytoma and glioblastoma patients stratified according to histological diagnosis within the individual groups defined by the IDH1 mutation status in combination with the MGMT promoter methylation status. 


\section{figure 6}
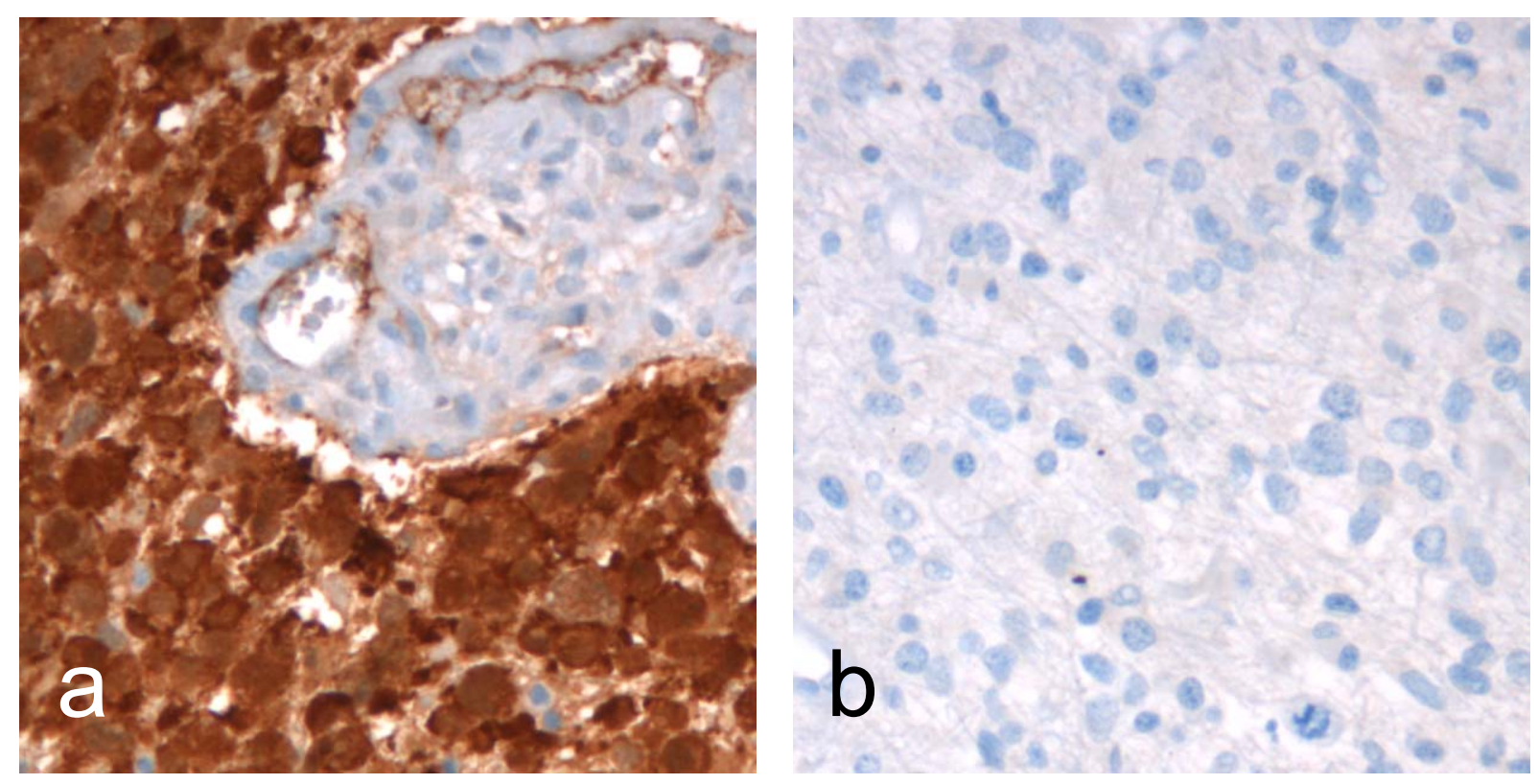

Representative images of a primary glioblastoma with IDH1R132H mutation detected by immunohistochemistry with monoclonal antibody H09 (panel a, left), and of an anaplastic astrocytoma without IDH1 mutation (panel b, right). Original magnification x200. 